ARTICLE

\title{
Electronegativity determination of individual surface atoms by atomic force microscopy
}

Jo Onoda ${ }^{1,2}$, Martin Ondráček ${ }^{3}$, Pavel Jelínek ${ }^{3,4}$ \& Yoshiaki Sugimoto ${ }^{1,2}$

Electronegativity is a fundamental concept in chemistry. Despite its importance, the experimental determination has been limited only to ensemble-averaged techniques. Here, we report a methodology to evaluate the electronegativity of individual surface atoms by atomic force microscopy. By measuring bond energies on the surface atoms using different tips, we find characteristic linear relations between the bond energies of different chemical species. We show that the linear relation can be rationalized by Pauling's equation for polar covalent bonds. This opens the possibility to characterize the electronegativity of individual surface atoms. Moreover, we demonstrate that the method is sensitive to variation of the electronegativity of given atomic species on a surface due to different chemical environments. Our findings open up ways of analysing surface chemical reactivity at the atomic scale.

\footnotetext{
${ }^{1}$ Department of Advanced Materials Science, Graduate School of Frontier Sciences, University of Tokyo, 5-1-5 Kashiwanoha, Kashiwa, Chiba 277-8561, Japan. ${ }^{2}$ Division of Electrical, Electronic and Information Engineering, Graduate School of Engineering, Osaka University, 2-1 Yamada-Oka, Suita, Osaka 565-0871, Japan. ${ }^{3}$ Department of Thin Films and Nanostructures, Institute of Physics, Czech Academy of Sciences, Cukrovarnická 10/112, Prague 16200, Czech Republic. ${ }^{4}$ Regional Centre of Advanced Technologies and Materials, Department of Physical Chemistry, Palacky University, Šlechtitelů 27, 78371 Olomouc, Czech Republic. Correspondence and requests for materials should be addressed to J.O. (email: jonoda@afm.k.u-tokyo.ac.jp).
} 
E lectronegativity, an important theoretical concept in chemistry, was originally defined by Linus Pauling as 'the power of an atom in a molecule to attract electrons to itself ${ }^{1,2}$. Since that time, various classical scales of electronegativity have been suggested ${ }^{3-6}$, including the rigorous modern formalism of absolute electronegativity developed along with density functional theory (DFT) $)^{7}$. In contrast, conventional experimental means for measuring electronegativity have been limited to thermochemical techniques, that is, the measurement of ensemble-averaged bond energies ${ }^{8}$. On the other hand, atomic force microscopy (AFM) was able to achieve atomic resolution on both semiconductor ${ }^{9}$ and insulator ${ }^{10}$ surfaces. It has also found numerous applications in the field of chemistry: for example, quantitative measurement of short-range chemical forces ${ }^{11}$, chemical identification of individual surface atoms ${ }^{12}$, visualization of the internal structures of organic molecules ${ }^{13-16}$ and metal clusters ${ }^{17}$, discrimination of the Pauling bond $\operatorname{order}^{18,19}$ and tracking of surface chemical reactions ${ }^{20,21}$.

Here, we extend this already impressive suite of applications by demonstrating the use of AFM in characterizing the electronegativity of individual surface atoms. Pauling's electronegativity values for individual single atoms on surfaces can be estimated using site-specific energy spectroscopy acquired with a variety of AFM tips. Namely, we show that the binding energies for individual surface atoms observed using different tips can provide an ensemble of data that can be used later to determine the electronegativity of different chemical elements or chemical groups on a surface (Fig. 1a). Our experimental findings are supported by theoretical analysis based on DFT calculations.

\section{Results}

Scatter plots of the bond energies. We demonstrate the concept using a prototypical $\mathrm{Si}(111)-(7 \times 7)$ surface with different local impurities (Supplementary Figs 1-5; Supplementary Methods 1). To characterize the Pauling electronegativity, we acquired bond energies by detecting the frequency shift $(\Delta f)$ from the resonant frequency $\left(f_{0}\right)$ of an oscillated $\mathrm{Si}$ cantilever at room temperature (Methods). As an example, we show tip-surface distance dependences of $\Delta f(\Delta f(z)$ curves) measured on $\mathrm{Si}$ and $\mathrm{O}$ adatoms on the $\mathrm{Si}(111)-(7 \times 7)$ surface in Fig. $1 \mathrm{~b}$. As seen in the inset of Fig. 2d, locally formed silicon oxide has a tetrahedral $\mathrm{SiO}_{4}$ structure, which is typical for the basic unit of silicate and stable even at room temperature ${ }^{22}$. By converting the $\Delta f(z)$ curves to a short-range energy-distance curves $(E(z)$; see Methods), we were able to obtain minimum potential energies (bond energies) on Si $\left(E_{\text {tip }-S i}\right)$ and $\mathrm{O}\left(E_{\text {tip }-\mathrm{O}}\right)$ as shown in Fig. 1c. Note that $E_{\text {tip }-\mathrm{O}}$ represents one of the strongest single polar covalent bonds $\sim 3 \mathrm{eV}$. Once the tip apex was deliberately changed by a tip-modification method, for example, mild contact with the sample surface, the magnitudes of $E_{\mathrm{tip}-\mathrm{Si}}$ and $E_{\mathrm{tip}-\mathrm{O}}$ were altered. This tip preparation method ensures that the tip apex would most likely be made of Si. Next, we investigated various sets of $E_{\text {tip }}-\mathrm{Si}$ and $E_{\text {tip - o }}$ by changing the tip state and then made a scatter plot of the bond energies as shown in Fig. 2d. Similarly, we investigated scatter plots of the bond energies associated with $\mathrm{Ge}, \mathrm{Sn}$ and $\mathrm{Al}$ adatoms on the $\mathrm{Si}(111)-(7 \times 7)$ surface (Fig. $2 \mathrm{a}-\mathrm{c})$. Remarkably, all the scatter plots showed linear relations. Fitting with a straight line, we were able to extract the slopes and intercepts as summarized in Tables 1 and 2.

Interpretation of the linear relations by Pauling's equation. We rationalized the linear relation between the bond energy of tip apexes and different surface atoms by employing Pauling's equation for the bond energy $E_{\mathrm{A}-\mathrm{B}}$ of heterogeneous polar bonds between atoms $A$ and $B^{1,2}$ :

$$
E_{\mathrm{A}-\mathrm{B}}=\sqrt{E_{\mathrm{A}-\mathrm{A}} E_{\mathrm{B}-\mathrm{B}}}+\Delta_{\mathrm{A}-\mathrm{B}}
$$

where $E_{\mathrm{A}-\mathrm{A}}\left(E_{\mathrm{B}-\mathrm{B}}\right)$ represents the energy of homogeneous bond $\mathrm{A}-\mathrm{A}(\mathrm{B}-\mathrm{B}) ; \sqrt{E_{\mathrm{A}-\mathrm{A}} E_{\mathrm{B}-\mathrm{B}}}$ and $\Delta_{\mathrm{A}-\mathrm{B}}$ are covalent and ionic energy parts of $E_{\mathrm{A}-\mathrm{B}}$, respectively. Thus, based on heterogeneous bond energies between the tip-termination atom and target $\mathrm{X}\left(E_{\text {tip }}-\mathrm{X}\right)$ and reference $\mathrm{Si}\left(E_{\text {tip-Si }}\right)$ atoms on the surface, which were experimentally obtained using the energy spectroscopy as described above, we deduced the following equation (Supplementary Note 1):

$$
E_{\text {tip }-\mathrm{X}}=\sqrt{\frac{E_{\mathrm{X}-\mathrm{X}}}{E_{\mathrm{Si}-\mathrm{Si}}}} E_{\text {tip }-\mathrm{Si}}+\Delta_{\text {tip }-\mathrm{X}} \text {. }
$$

The homogeneous bond energy $E_{\mathrm{X}-\mathrm{X}}\left(E_{\mathrm{Si}-\mathrm{Si}_{\mathrm{i}}}\right)$ will be discussed next using theoretical models. According to the Pauling theory, the term $\Delta_{\text {tip - x }}$ represents the ionic energy stemming from the difference in electronegativity between the tip and the target atom X. In this context, the linear dependence observed in Fig. 2 can be interpreted as an experimental confirmation of the validity of Pauling's equation (1) for the electronegativity scale.

Chemical identification by the slopes. Table 1 shows experimentally determined slopes in the scatter plots of the bond energies. Interestingly, the slope for the Ge and Sn adatoms match the interaction ratios of the maximum attractive forces of the same atomic species determined previously ${ }^{12,23,24}$. As discussed in ref. 12, the interaction ratio of the maximal forces (or similarly the minimum energies) represents the 'fingerprint' of the chemical identities. If there is no electronegativity difference between the tip and the target atom $\mathrm{X}$, the term $\Delta_{\text {tip - } \mathrm{X}}$ in equation (2) vanishes and the following a

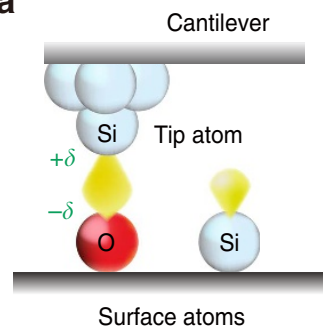

b

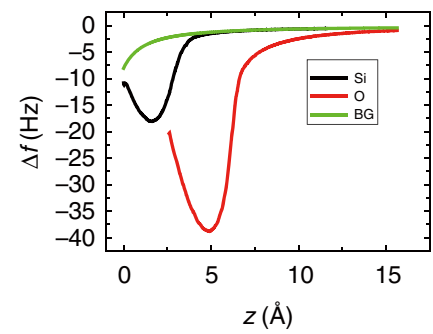

c

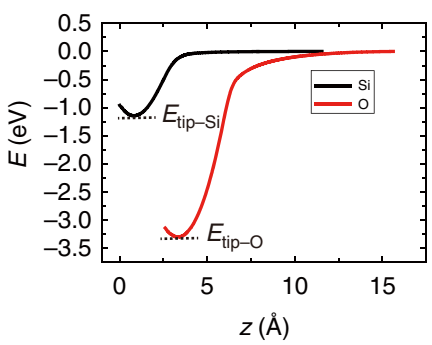

Figure 1 | Site-specific energy spectroscopy. (a) Schematic illustration of AFM energy spectroscopy with the polar covalent bond of Si-O. (b) $\Delta f$ curves measured on $\mathrm{Si}$ and $\mathrm{O}$ adatoms on the $\mathrm{Si}(111)-(7 \times 7)$ surface. The background $(B G) \Delta f(z)$ curve is also included for subtraction of the long-range component (Methods). (c) $E(z)$ curves converted from short-range components in the $\Delta f(z)$ curves in $\mathbf{b}$. Bond energies are defined at the minima of $E(z)$ curves. The acquisition parameters are $f_{0}=152.913 \mathrm{kHz}$, the oscillation amplitude $(A)=146 \AA$, the spring constant $(\mathrm{k})=28.2 \mathrm{~N} \mathrm{~m}^{-1}$ and sample bias $\left(V_{\mathrm{s}}\right)=+40 \mathrm{mV}$. 

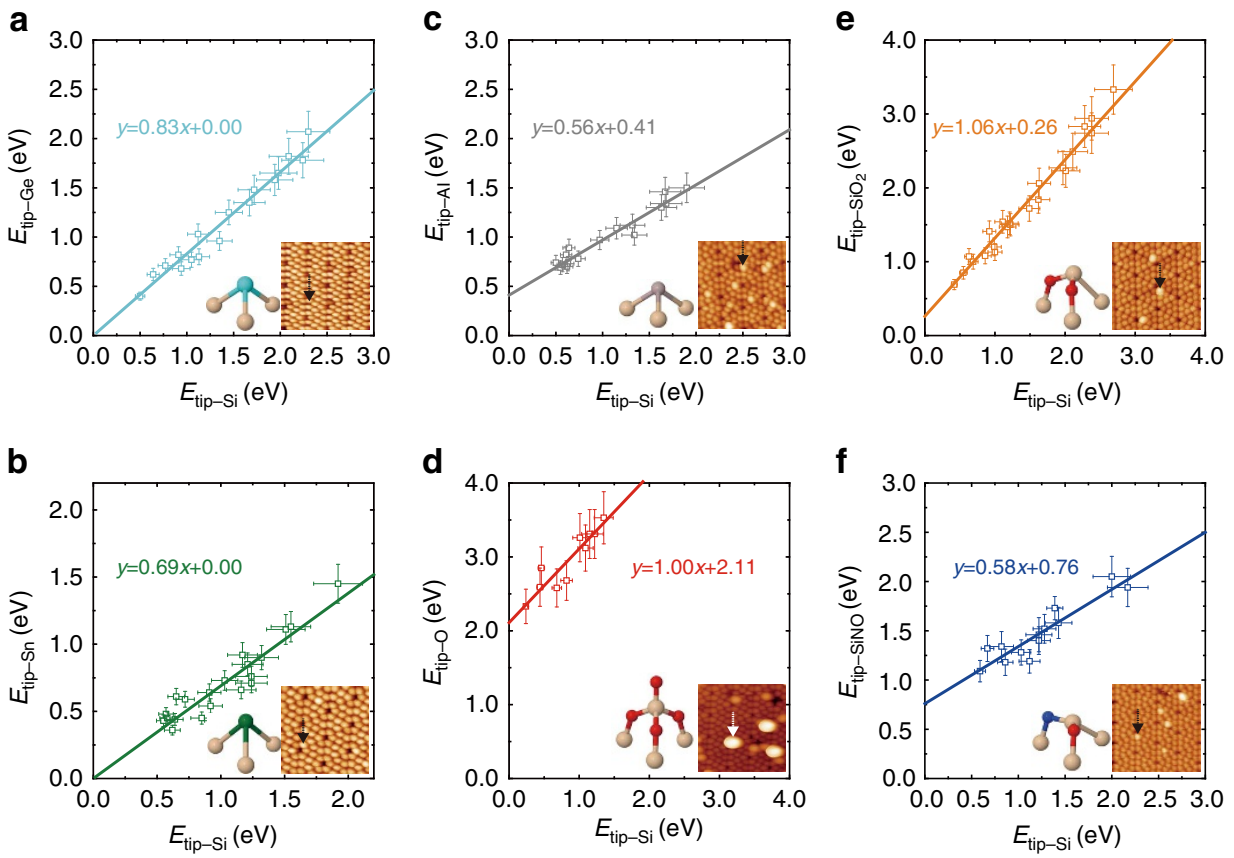

Figure 2 | Linear relations between bond energies of different chemical species. Scatter plots of the bond energies of $\mathrm{Ge}(\mathbf{a}), \mathrm{Sn}(\mathbf{b}), \mathrm{Al}(\mathbf{c})$, $\mathrm{O}(\mathbf{d})$ adatoms, $\mathrm{SiO}_{2}(\mathbf{e})$ and $\mathrm{SiNO}(\mathbf{f})$ complexes obtained experimentally. The bond energies were measured above the bright spots in AFM images as shown in the insets. The individual error bars are estimated as $10 \%$ of the corresponding bond energies based on uncertainties in measurements of $A$ and $k$. The scatter plots were fitted using weighted orthogonal distance regression.

\begin{tabular}{|c|c|c|c|}
\hline Bond configuration & Slope (exp.) & Slope (cal.) & $E_{\mathbf{X}-\mathbf{x}}$ by DFT (eV) \\
\hline $\mathrm{Si}-\mathrm{Si}$ & 1 & 1 & 2.20 \\
\hline $\mathrm{Ge}-\mathrm{Ge}$ & $0.83 \pm 0.07$ & 0.83 & 1.52 \\
\hline Sn-Sn & $0.69 \pm 0.07$ & 0.76 & 1.27 \\
\hline Al-Al & $0.56 \pm 0.07$ & 0.46 & 0.47 \\
\hline $\mathrm{O}-\mathrm{O}$ & $1.00 \pm 0.26$ & $0.89^{\star}$ & - \\
\hline $\mathrm{O}_{2} \mathrm{Si}-\mathrm{SiO}_{2}$ & $1.06 \pm 0.07$ & 1.17 & 2.99 \\
\hline ONSi-SiNO & $0.58 \pm 0.11$ & 0.41 & 0.37 \\
\hline
\end{tabular}

relation holds: $E_{\text {tip }-\mathrm{X}} / E_{\mathrm{tip}-\mathrm{Si}}=\sqrt{E_{\mathrm{X}-\mathrm{X}} / E_{\mathrm{Si}-\mathrm{Si}}}$. To verify this relation, we calculated the homogeneous bond energies $E_{\mathrm{X}}-\mathrm{X}$ and the related theoretical slopes (Table 1) for various elements (including $E_{\mathrm{Si}-\mathrm{Si}}$ ) by modelling the interactions between simple mirror-geometry clusters (Supplementary Fig. 6; Supplementary Methods 2). Notably, all calculated values reproduced very well the experimental slopes. Thus, the values of slopes in the scatter plots characterize the covalent bond energy of target $\mathrm{X}$ adatoms with tip renormalized by the $E_{\mathrm{Si}-\mathrm{Si}}$ bonding energy. We should stress that the previous chemical identification method $^{12}$ holds only for elements having very similar electronegativity. By contrast, the present identification method based on scatter plots of the bond energies is applicable to arbitrary elements with divergent electronegativity.

Electronegativity determination by the intercepts. Equation (2) indicates that the intercepts in the scatter plots of the bond energies can be interpreted as the ionic energies, which are correlated with electronegativity values of the tip $\left(\chi_{\text {tip }}\right)$ and the $\mathrm{X}\left(\chi_{\mathrm{X}}\right)$ atoms through the equation of $\Delta_{\text {tip }-\mathrm{X}}=1.3\left(\chi_{\text {tip }}-\chi_{\mathrm{X}}\right)^{2}$ (refs 1,2). Since atomically sharp tips were prepared by

\begin{tabular}{|c|c|c|c|c|}
\hline Target $\mathbf{X}$ & Intercept (eV) & $\left|\chi_{\mathbf{S i}}-\chi_{\mathbf{X}}\right|$ & $\chi_{x}$ & Pauling's $\chi_{x}$ \\
\hline $\mathrm{Si}$ & - & - & $\begin{array}{c}1.8 \\
\text { (as reference) }\end{array}$ & 1.8 \\
\hline $\mathrm{Ge}$ & $0.00 \pm 0.07$ & $\sim 0$ & $\sim 1.8$ & 1.8 \\
\hline Sn & $0.00 \pm 0.06$ & $\sim 0$ & $\sim 1.8$ & 1.8 \\
\hline Al & $0.41 \pm 0.06$ & $0.56 \pm 0.04$ & $1.24 \pm 0.04$ & 1.5 \\
\hline O & $2.11 \pm 0.22$ & $1.27 \pm 0.07$ & $3.07 \pm 0.07$ & 3.5 \\
\hline $\mathrm{SiO}_{2}$ & $0.26 \pm 0.08$ & $0.45 \pm 0.07$ & $2.25 \pm 0.07$ & - \\
\hline SiNO & $0.76 \pm 0.12$ & $0.77 \pm 0.06$ & $2.57 \pm 0.06$ & - \\
\hline
\end{tabular}

intentional poking into clean regions of the Si surfaces, we assume that their apexes are terminated with $\mathrm{Si}$ atoms such that individual $\chi_{\text {tip }}$ are almost the same magnitude as that of an $\mathrm{Si}$ adatom $\left(\chi_{\mathrm{Si}}\right)$ on the $\operatorname{Si}(111)-(7 \times 7)$ surface. This assumption is justified by the experimental facts that all the scatter plots in Fig. 2 demonstrate the linear relation (Supplementary Figs 7 and 8; Supplementary Note 2), and by theoretical calculations, in which $\mathrm{Si}$-tip models mimicking a surface $\mathrm{Si}$ adatom can provide good agreement with experimental evidences ${ }^{25}$. We find the total-energy DFT calculations simulating few Si-tip models (Supplementary Fig. 9) interacting with the $\operatorname{Si}(111)-7 \times 7$ slab surface can reproduce well bond energies regarding with Ge, $\mathrm{Al}$ and $\mathrm{SiO}_{2}$ (discussed later) acquired with tips having large $E_{\mathrm{Si}-\mathrm{Si}}$ bonding energy $>1.0 \mathrm{eV}$ (Supplementary Fig. 10). However, slopes and intercepts estimated only with the theoretical plots do not reproduce exactly the experimental values. We attributed this discrepancy to statistical uncertainty due to very small number of calculated points corresponding to only five highly reactive Si-tip models (for more details, see Supplementary Note 3). 
We should note, from the equation of $\Delta_{\mathrm{Si}-\mathrm{X}}=1.3\left(\chi_{\mathrm{Si}}-\chi_{\mathrm{X}}\right)^{2}$, it is evident that only difference between electronegativities of the reference Si tip $\left(\chi_{\mathrm{Si}}\right)$ and target $\mathrm{X}\left(\chi_{\mathrm{X}}\right)$ can be directly determined within the experimental precision. Thus, we need calibration of the reference electronegativity $\left(\chi_{\mathrm{Si}}\right)$ for a quantitative comparison of obtained electronegativity values with those tabulated in the periodic table. Throughout this report, we deliberately assume $\chi_{\mathrm{Si}}=1.8$ in accordance with the definition in Pauling's electronegativity scale $\mathrm{e}^{1,2}$.

To determine the electronegativity values of individual target $\mathrm{X}$ adatoms from the relation $\Delta_{\mathrm{Si}-\mathrm{X}}=1.3\left(\chi_{\mathrm{Si}}-\chi_{\mathrm{X}}\right)^{2}$, we have to know the sign of the electronegativity differences $\left(\left|\chi_{\mathrm{Si}}-\chi_{\mathrm{X}}\right|\right.$ in Table 2). This information is not a priori accessible from the present experimental approach. One possibility is to stick with a 'chemical intuition', when the chemical origin of the impurities is known (for example, $\mathrm{Al}$ is less electronegative than $\mathrm{Si}$ ). Another one is 'classical' approach to compare a scatter plot obtained by $\mathrm{Si}$ tips with another plot acquired by chemically different (such as Al-terminated) tips whose electronegativity is diverged from $\chi_{\mathrm{Si}}$. We expect that future work with at least two chemically different tips would give us a clue for the sign of electronegativity differences. For an alternative approach, we consider the modern definition of electronegativity ${ }^{26}$, where the electronegativity is directly related to the chemical potential $\mu$ (that is, work function): $\mu=(\partial E / \partial N)=-\chi$, where $E$ and $N$ represent the total electronic energy and number of electrons, respectively. In the case of surfaces, the electronegativity $\chi$ can be replaced with a local variation of the work function on a given surface site. The variation of the work function on surfaces with atomic-scale resolution can be estimated by Kelvin probe force microscopy $(\mathrm{KPFM})^{27}$. Unfortunately, the presence of artefacts at close tip-sample distances hampers the reliable interpretation of a KPFM signal at the minimum potential energy ${ }^{28}$. Thus, here, we opted for a theoretical evaluation of the local work functions analysing the Hartree potential $\left(V_{\mathrm{H}}(z)\right)$ obtained from DFT calculations. We believe, however, that the advancement of KPFM should allow us to determine the sign of the electronegativity difference exclusively by experimental measurements in the near future.

Total-energy DFT simulations reveal that the adatoms of various elements on a $\mathrm{Si}(111)-(7 \times 7)$ surface show variations of local density of states (Supplementary Fig. 11) and electron density (Supplementary Fig. 12) near the Fermi energy, and of $V_{\mathrm{H}}(z)$ above the inspected adatom (Supplementary Fig. 13). Figure 3 a displays the calculated one-dimensional profiles of $V_{\mathrm{H}}(z)$ along the $z$ axis, representing the local work functions above the $\mathrm{Si}, \mathrm{Ge}, \mathrm{Sn}, \mathrm{Al}$ and $\mathrm{O}$ adatoms. Interestingly, the local work functions at a $z$-distance of 3-4 $\AA$ above Ge and $\mathrm{Sn}$ adatoms are very similar to that of a $\mathrm{Si}$ adatom. This observation together with the lack of an intercept in the experimental scatter plots of Ge and Sn (Fig. 2a,b) apparently confirms the above assumption that $\chi_{\text {tip }}$ is close to $\chi_{\mathrm{Si}}$; that is, $\chi_{\mathrm{Ge}}=\chi_{\mathrm{Sn}}=\chi_{\mathrm{Si}}=1.8$ (Table 2). On the other hand, the local work functions of $\mathrm{Al}$ and $\mathrm{O}$ show effectively lower and higher values, respectively, than the $\mathrm{Si}$ adatom shown in Fig. 3a. This means $\chi_{\mathrm{Al}}<\chi_{\mathrm{Si}}$ and $\chi_{\mathrm{O}}>\chi_{\mathrm{Si}}$, and the electronegativities of $\mathrm{Al}$ and $\mathrm{O}$ become $1.24 \pm 0.04$ and $3.07 \pm 0.07$, respectively. These values are in very good agreement with Pauling's electronegativities (Table 2).

Evaluation of group electronegativity. In general, the electronegativity of surface atoms (here, Si adatoms) can be modified by their surrounding environments: for example, structural and chemical rearrangement of local structures, charge transfer from the neighbouring atoms and re-hybridization of orbitals. Previously, this information has not been experimentally accessible. From this perspective, the present method provides a unique opportunity to obtain electronegativity values for specific surface atoms in different local chemical structures. To demonstrate such sensitivity towards group electronegativity, we carried out measurements on a ' $\mathrm{SiO}_{2}$ ' structure (Fig. 2e), where two $\mathrm{O}$ atoms are inserted into the back bonds of an $\mathrm{Si}$ adatom ${ }^{22}$, and on a 'SiNO' structure (Fig. 2f) created from dissociated $\mathrm{O}$ and $\mathrm{N}$ atoms (Supplementary Methods 1). Local atomic arrangements obtained from the total-energy DFT calculations of the $\mathrm{SiO}_{2}$ and SiNO structures are presented in Fig. 3c,d, respectively.

Although the topmost atom of the $\mathrm{SiO}_{2}$ configuration is $\mathrm{Si}$, the scatter plot of bond energies for $\mathrm{SiO}_{2}$ (Fig. 2e) clearly shows an intercept of $0.26 \pm 0.08 \mathrm{eV}$. To understand the presence of the intercept, we analysed the variation of $V_{\mathrm{H}}(z)$ for $\mathrm{SiO}_{2}$. Figure 3a,e,f shows that the local work function above the $\mathrm{Si}$ adatom in the $\mathrm{SiO}_{2}$ configuration is higher than that for the pure $\mathrm{Si}$ adatom, that is, $\chi_{\mathrm{SiO}_{2}}>\chi_{\mathrm{Si}}$. Consequently, we can estimate the electronegativity of the $\mathrm{Si}$ adatom in $\mathrm{SiO}_{2}$ to be $2.25 \pm 0.07$, and a

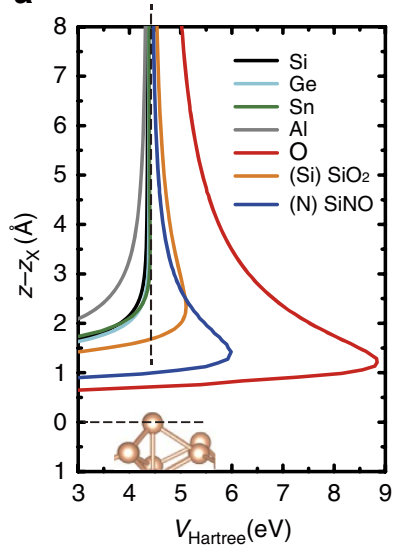

b

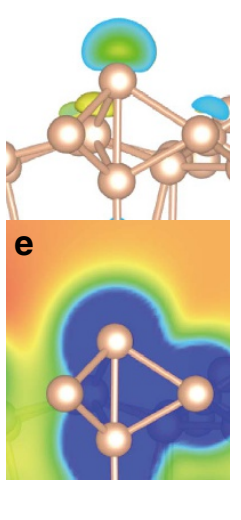

C

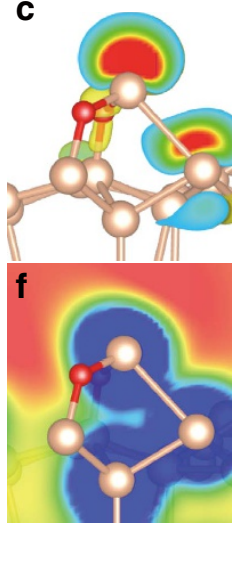

d

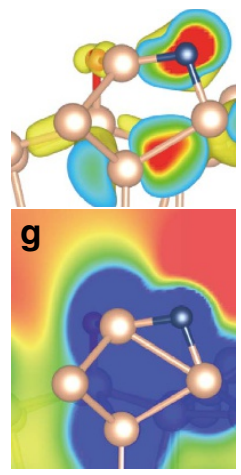

Figure 3 | Evaluation of electronegativity difference by DFT calculations. (a) A one-dimensional plot showing the variation of the Hartree potential above different adatoms such as $\mathrm{Si}, \mathrm{Ge}, \mathrm{Al}, \mathrm{O}$ or $\mathrm{N}$. The zero point of the Hartree potential is set to the Fermi level, and the $z$-distance is aligned to the height of an inspected adatom $\left(z_{X}\right)$ on the $\mathrm{Si}(111)-(7 \times 7)$ surface. The calculated electron density isosurfaces $(0.02$ e $\AA-3$ in yellow) of the highest occupied frontiers orbital on the $\mathrm{Si}$ adatom (b), $\mathrm{SiO}_{2}(\mathbf{c})$ around the Fermi level and $\mathrm{SiNO}$ (d) located 1 eV below the Fermi level. Cut-plane of the electron density isosurface is coloured from red $\left(0.13\right.$ e $\left.\AA^{-3}\right)$ to blue $\left(0.02\right.$ e $\left.\AA^{-3}\right)$. Plotted two-dimensional plane of the Hartree potential projected onto the Si adatom (e), $\mathrm{SiO}_{2}$ (f) and $\mathrm{SiNO}(\mathbf{g})$ complex (range from $\mathrm{O}$ (blue) to 5 (red) eV). 
thus more electronegative than that of pure Si (Table 2). It is worth noting that while the intercept appears, the slope remains close to 1 . Thus, it is evident that the local charge transfer in the $\mathrm{SiO}_{2}$ structure between the $\mathrm{Si}$ adatom and adjacent $\mathrm{O}$ atoms changes the ionic energy term $\Delta$ but leaves the covalent bond energy almost unaffected. This effect makes sense given that the dangling bond on the $\mathrm{Si}$ adatom of $\mathrm{SiO}_{2}$ maintains an $s p_{3}$ hybridization very similar to that of an $\mathrm{Si}$ adatom on a clean surface (Fig. 3b,c), but with a different charge density.

The case of SiNO is more complex. Experimentally, the scatter plot of bond energies for SiNO (Fig. 2f) shows an intercept of $0.76 \pm 0.12 \mathrm{eV}$. The magnitude of the experimental slope is far from unity, as is the theoretical slope for SiNO (Table 1). This reveals that the atomic and electronic structure of the SiNO complex differs from that of a bare $\mathrm{Si}$ adatom and/or that the neighbouring $\mathrm{N}$ atom as well as the $\mathrm{Si}$ atom is involved in the chemical interaction with the $\mathrm{Si}$ atom at the tip apex. Indeed, according to the DFT calculations the atomic structure of the SiNO complex is different from the $\mathrm{Si}$ adatom, as shown in Fig. 3b,d. The highest occupied frontier orbital of the SiNO structure is delocalized between the $\mathrm{Si}, \mathrm{N}$ and $\mathrm{O}$ atoms (Fig. $3 \mathrm{~d}$ ). Therefore, one should consider the whole structure instead of individual atoms in the electronegativity analysis. To determine the sign of $\left|\chi_{\mathrm{Si}}-\chi_{\mathrm{SiNO}}\right|$, we analysed the spatial distribution of $V_{\mathrm{H}}(z)$ over the SiNO structure. Figure 3g shows the result: the work function is maximal at the $\mathrm{N}$ atom of SiNO (Fig. 3a), and monotonically decreases towards the $\mathrm{Si}$ atom but still remains slightly higher at the relevant $z$-distance than it is over the pure $\mathrm{Si}$ adatom; hence, $\chi_{\mathrm{SiNO}}>\chi_{\mathrm{Si}}$. Accordingly, we determined the experimental electronegativity of the SiNO structure to be $2.57 \pm 0.06$. Compared to the conventional Pauling's electronegativities of $\mathrm{Si}(1.8)$ and $\mathrm{N}(3.0)$, the obtained electronegativity of the SiNO structure reflects its intermediate character. In sum, the analysis of the $\mathrm{SiNO}$ and $\mathrm{SiO}_{2}$ complexes indicates that AFM is able to determine the group electronegativity.

In conclusion, we have presented here a method that, for the first time to our knowledge, allows experimental characterization of the electronegativity of individual surface atoms by means of AFM. We have found that the scatter plots of the bond energies can be interpreted based on Pauling's equation for polar covalent bonds. This allowed us to disentangle the covalent and ionic bond energies in polar covalent bonds, and hence to estimate the electronegativity of the individual elements. In addition, this method facilitates analysis of the group electronegativity of atoms belonging to locally formed nanostructures. We expect that the present method can be applied to other interesting systems such as transition metal oxides used in catalysis by terminating AFM tips with known atoms on the surface. Furthermore, we believe that the method can not only characterize the specific electronegativity of individual atoms, defects, adsorbates and dopant impurities on surfaces but may also open up new ways of analysing surface chemical reactivity in terms of surface electrophilicity/nucleophilicity against reactant ${ }^{29}$, and surface chemical softness/hardness with regard to the hard-soft acid-base principle ${ }^{26,30}$.

\section{Methods}

Atomic force microscopy. All experiments were carried out using a custom-built ultrahigh-vacuum AFM system at room temperature with a typical base pressure of $5 \times 10^{-9} \mathrm{~Pa}$. An optical interferometer was equipped to detect the cantilever deflection. Frequency-modulation technique was used for obtaining $\Delta f$. We used commercial Si cantilevers, whose tip apexes were cleaned by Ar ion sputtering to remove the native oxidation layers. The cantilever was oscillated with sufficiently large amplitude $(A \sim 100 \AA)$ for stable AFM operation. We chose the $\mathrm{Si}(111)-(7 \times 7)$ surface as playground for the purpose of the present experiments since the surface had many $\mathrm{Si}$ adatoms on which atoms at tip apex were replaced by mild contact. To compensate a contact potential difference between tip
}

and sample, we properly applied $V_{\mathrm{S}}$ to the sample while keeping the tip bias at ground.

Site-specific energy spectroscopy. When we performed site-specific energy spectroscopy, thermal drift was compensated by feed-forward technique ${ }^{31}$ and the precision of lateral position of the point spectroscopy became better than $\pm 0.1 \AA$, both of which were realized by our atom-tracking implementation ${ }^{32}$. After recording several $\Delta f(z)$ curves on an adatom of interest, we averaged them to a single $\Delta f(z)$ curve for converting to $E(z)$. Note that energy dissipation during the spectroscopy was negligible. For eliciting accurate bond energies exerted on the foremost tip atom and surface adatoms, we subtracted the background $\Delta f(z)$ curve (Fig. 1b; Supplementary Methods 1), which is obtained by analytically fitting a $\Delta f(z)$ curve measured on a corner hole site ${ }^{11,12}$, from $\Delta f(z)$ curves on the individual target and reference adatom sites. Finally, the $\Delta f(z)$ curves only containing the short-range contribution were converted to $E(z)$ using the inversion procedure suggested by Sader and Jarvis ${ }^{33}$.

Density functional theory calculations. All DFT calculations have been done using the Vienna Ab-Initio Simulation Package (VASP 4.6$)^{34,35}$, which is a pseudopotential plane-wave code. The basic versions of ultrasoft Vanderbilt pseudopotentials ${ }^{36,37}$ supplied with VASP were used for all chemical elements involved ( $\mathrm{Si}, \mathrm{H}, \mathrm{O}, \mathrm{N}, \mathrm{Al}$ and $\mathrm{Ge}$ ) apart from $\mathrm{Sn}$, for which the Snd potential that includes $d$-electrons as valence electrons was adopted. The PW91 implementation of the generalized gradient approximation ${ }^{38}$ was chosen to describe the exchangecorrelation functional. The size of the plane-wave basis was determined by the cutoff energy of 300 or $396 \mathrm{eV}$. Only the central (Gamma) point of the first Brillouin zone was considered for the Bloch-wave solutions.

Data availability. The data that support the findings of this study are available from the corresponding author on reasonable request.

\section{References}

1. Pauling, L. The nature of the chemical bond. IV. The energy of single bonds and the relative electronegativity of atoms. J. Am. Chem. Soc. 54, 3570-3582 (1932).

2. Pauling, L. in The Nature of the Chemical Bond 3rd, 13th Printing 1995 edn (Cornell University Press, 1960).

3. Mulliken, R. S. A new electroaffinity scale; together with data on valence states and on valence ionization potentials and electron affinities. J. Chem. Phys. 2, 782-793 (1934).

4. Sanderson, R. T. Electronegativities in inorganic chemistry. J. Chem. Educ. 29, 539-544 (1952).

5. Allred, A. L. \& Rochow, E. G. A scale of electronegativity based on electrostatic force. J. Inorg. Nucl. Chem. 5, 264-268 (1958).

6. Gordy, W. A new method of determining electronegativity from other atomic properties. Phys. Rev. 69, 604-607 (1946).

7. Parr, R. G., Donnelly, R. A., Levy, M. \& Palke, W. E. Electronegativity: the density functional viewpoint. J. Chem. Phys. 68, 3801-3807 (1978).

8. Wagman, D. D. et al. in The NBS Tables of Chemical Thermodynamic Properties: Selected Values for Inorganic and C1 and C2 Organic Substances in SI Units (American Chemical Society and American Institute of Physics, 1982).

9. Giessibl, F. J. Atomic resolution of the silicon $(111)-(7 \times 7)$ surface by atomic force microscopy. Science 267, 68 (1995).

10. Barth, C. \& Reichling, M. Imaging the atomic arrangements on the hightemperature reconstructed $\alpha-\mathrm{Al}_{2} \mathrm{O}_{3}(0001)$ surface. Nature 414, $54-57$ (2001).

11. Lantz, M. A. et al. Quantitative measurement of short-range chemical bonding forces. Science 291, 2580-2583 (2001).

12. Sugimoto, Y. et al. Chemical identification of individual surface atoms by atomic force microscopy. Nature 446, 64-67 (2007).

13. Gross, L., Mohn, F., Moll, N., Liljeroth, P. \& Meyer, G. The chemical structure of a molecule resolved by atomic force microscopy. Science 325, 1110-1114 (2009).

14. Gross, L. et al. Organic structure determination using atomic-resolution scanning probe microscopy. Nat. Chem. 2, 821-825 (2010).

15. Mohn, F., Gross, L., Moll, N. \& Meyer, G. Imaging the charge distribution within a single molecule. Nat. Nanotechnol. 7, 227-231 (2012).

16. Iwata, K. et al. Chemical structure imaging of a single molecule by atomic force microscopy at room temperature. Nat. Commun. 6, 7766 (2015).

17. Emmrich, M. et al. Subatomic resolution force microscopy reveals internal structure and adsorption sites of small iron clusters. Science 348, 308-311 (2015).

18. Gross, L. et al. Bond-order discrimination by atomic force microscopy. Science 337, 1326-1329 (2012)

19. Pavliček, N. et al. On-surface generation and imaging of arynes by atomic force microscopy. Nat. Chem. 7, 623-628 (2015).

20. de Oteyza, D. G. et al. Direct imaging of covalent bond structure in single-molecule chemical reactions. Science 340, 1434-1437 (2013). 
21. Schuler, B. et al. Reversible bergman cyclization by atomic force microscopy. Nat. Chem. 8, 220-224 (2016).

22. Onoda, J., Ondráček, M., Yurtsever, A., Jelínek, P. \& Sugimoto, Y. Initial and secondary oxidation products on the $\mathrm{Si}(111)-(7 \times 7)$ surface identified by atomic force microscopy and first principle calculations. Appl. Phys. Lett. 104, 133107 (2014)

23. Setvín, M. et al. Chemical identification of single atoms in heterogeneous III-IV chains on $\mathrm{Si}(100)$ surface by means of nc-AFM and DFT calculations. ACS Nano 6, 6969-6976 (2012).

24. Onoda, J., Niki, K. \& Sugimoto, Y. Identification of Si and Ge atoms by atomic force microscopy. Phys. Rev. B 92, 155309 (2015).

25. Pou, P. et al. Structure and stability of semiconductor tip apexes for atomic force microscopy. Nanotechnology 20, 264015 (2009).

26. Parr, R. G. \& Pearson, R. G. Absolute hardness: companion parameter to absolute electronegativity. J. Am. Chem. Soc. 105, 7512-7516 (1983).

27. Sadewasser, S. \& Glatzel, T. Kelvin Probe Force Microscopy (Springer-Verlag, 2012).

28. Sadewasser, S. et al. New insights on atomic-resolution frequency-modulation Kelvin-probe force-microscopy imaging of semiconductors. Phys. Rev. Lett. 103, 266103 (2009).

29. Brommer, K. D., Galván, M., Pino, Jr A. D. \& Joannopoulos, J. D. Theory of adsorption of atoms and molecules on $\mathrm{Si}(111)-(7 \times 7)$. Surf. Sci. 314, $57-70$ (1994).

30. Pearson, R. G. Hard and soft acids and bases. J. Am. Chem. Soc. 85, 3533-3539 (1963).

31. Abe, M. et al. Drift-compensated data acquisition performed at room temperature with frequency modulation atomic force microscopy. Appl. Phys. Lett. 90, 203103 (2007).

32. Abe, M., Sugimoto, Y., Custance, O. \& Morita, S. Room-temperature reproducible spatial force spectroscopy using atom-tracking technique. Appl. Phys. Lett. 87, 173503 (2005).

33. Sader, J. E. \& Jarvis, S. P. Accurate formulas for interaction force and energy in frequency modulation force spectroscopy. Appl. Phys. Lett. 84, 1801-1803 (2004).

34. Kresse, G. \& Furthmüller, J. Efficiency of $a b$ initio total energy calculations for metals and semiconductors using a plane-wave basis set. Comput. Mater. Sci. 6, 15-50 (1996).

35. Kresse, G. \& Furthmüller, J. Efficient iterative schemes for ab initio totalenergy calculations using a plane-wave basis set. Phys. Rev. B 54, 11169-11186 (1996).

36. Vanderbilt, D. Soft self-consistent pseudopotentials in a generalized eigenvalue formalism. Phys. Rev. B 41, 7892-7895 (1990).

37. Kresse, G. \& Hafner, J. Norm-conserving and ultrasoft pseudopotentials for first-row and transition-elements. J. Phys.: Condens. Matter 6, 8245-8257 (1994).
38. Perdew, J. P. et al. Atoms, molecules, solids, and surfaces: applications of the generalized gradient approximation for exchange and correlation. Phys. Rev. B 46, 6671-6687 (1992).

\section{Acknowledgements}

This work was supported by Grants-in-Aid for JSPS Fellows (14J00689) and for Scientific Research $(15 \mathrm{H} 03566,16 \mathrm{H} 00933,16 \mathrm{H} 00959,16 \mathrm{~K} 13680,16 \mathrm{~K} 17482)$ from the Ministry of Education, Culture, Sports, Science, and Technology of Japan (MEXT). We also acknowledge financial support from the Asahi Glass Foundation, the Mitsubishi Foundation, the Yamada Science Foundation, Ube foundation, and the Precise Measurement Technology Promotion Foundation. P.J. and M.O. acknowledge financial support of GACR (Czech Science Foundation) under Project no. 14-16963J and Czech Academy of Sciences through Praemium Academiae award.

\section{Author contributions}

J.O. and Y.S. conceived and designed the experiments. J.O. and Y.S. performed the experiments. M.O. and P.J. performed the theoretical analysis. J.O. and P.J. wrote the paper. All authors discussed the results and commented on the manuscript.

\section{Additional information}

Supplementary Information accompanies this paper at http://www.nature.com/ naturecommunications

Competing interests: The authors declare no competing financial interests.

Reprints and permission information is available online at http://npg.nature.com/ reprintsandpermissions/

How to cite this article: Onoda, J. et al. Electronegativity determination of individual surface atoms by atomic force microscopy. Nat. Commun. 8, 15155 doi: $10.1038 /$ ncomms15155 (2017)

Publisher's note: Springer Nature remains neutral with regard to jurisdictional claims in published maps and institutional affiliations.

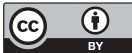

This work is licensed under a Creative Commons Attribution 4.0 International License. The images or other third party material in this article are included in the article's Creative Commons license, unless indicated otherwise in the credit line; if the material is not included under the Creative Commons license, users will need to obtain permission from the license holder to reproduce the material. To view a copy of this license, visit http://creativecommons.org/licenses/by/4.0/

C) The Author(s) 2017 